\title{
PARTITIONS BY REAL ALGEBRAIC VARIETIES, AND APPLICATIONS TO QUESTIONS OF INONLINEAR APPROXIMATION
}

\author{
BY HUGH E. WARREN
}

Communicated by J. Cesari, June 20, 1966

In recent investigations concerning Chebyshev approximation one has often considered in place of ordinary polynomials as the allowable approximating functions more general functions. For instance, many authors considered an arbitrary $\boldsymbol{n}$ dimensional linear vector space of continuous functions on some compact set, that is, a family which depends linearly on $n$ parameters, and obtained among other things lower bounds for the degree of approximation of certain function classes (see for example Lorentz [1]).

Vitushkin [5] obtained analogous results for more extended families in which the dependence on the parameters is polynomial of higher degree. (He also studied families in which the dependence on the parameters is rational, and even piecewise rational; it turns out that the methods developed for the polynomial case work also in these cases.) To be precise, a subset $S$ of any linear space $L$ is called a "family depending polynomially on $n$ parameters" if the general element of $S$ has the form $P\left(y_{1}, \cdots, y_{n}\right), y_{1}, \cdots, y_{n}$ in the base field of $L$, where $P\left(x_{1}, \cdots, x_{n}\right)$ is a polynomial in the indeterminates $x_{1}, \ldots, x_{n}$ with elements of $L$ as coefficients. If the polynomial degree of $P$ is $d, S$ is said to depend polynomially of degree $d$ on $n$ parameters. If $d$ equals one, $S$ is of course an $n$ dimensional vector space. It will be assumed below that $d$ is greater than one.

If in addition $L$ is a normed linear space with norm \|\| , one can speak of approximating an arbitrary family $F$ contained in $L$ by a family $S$ depending polynomially of degree $d$ on $n$ parameters. In such a case one says $S$ approximates $F$ to within $\delta$ if

$$
\sup _{x \in F} \inf _{y_{1}, \ldots, \nu_{n}}\left\|X-P\left(y_{1}, \cdots, y_{n}\right)\right\|=\delta .
$$

The above mentioned results of Vitushkin are lower bounds for $\delta$ in terms of $n$ and $d$ for certain families $F$ of continuous functions with the supremum norm.

The proofs of Vitushkin's results are quite complicated. The main tools are an estimate of the number of components of a real algebraic variety, due to Oleinik and Petrovsky [2], and results on "variations of sets" as expounded in Vitushkin [4], [5].

The present writer has obtained upper bounds for the partition of $n$-space by real algebraic surfaces sharper than those directly de- 
rivable from the work of Oleinik and Petrovsky; see \$1 below. Using these bounds, and adapting a technique of Shapiro [3], one can very easily prove the main results of Vitushkin (with sharper constants) without making any use whatsoever of "variations of sets" (\$2). The method used is easily modified to yield also the analogous results for $L^{p}$ approximation.

Insofar as the problem of partitioning space by real algebraic varieties has independent interest, $\$ 3$ gives further results concerning this problem, results of a precision beyond what is needed for applications to approximation theory.

1. A general result on the partitions of space by real varieties. Let $p_{1}, \cdots, p_{m}$ be real polynomials in $n$ variables, each of degree not exceeding $d$. Put $E=R^{n}-\bigcup_{i=1}^{m}\left\{p_{i}=0\right\}$, where $R^{n}$ denotes a real $n$ dimensional Euclidean space. The number of components of $E$ can be bounded by a function of $m, n$, and $d$. One has in particular

THEOREM 1. If $m \geqq 2 n$, the number of components of $E$ is less than $((4 e d m) / n)^{n}$.

COROLlaRY 1. The number of sign sequences $s=\left(s_{1}, \cdots, s_{m}\right)$, $s_{i}= \pm 1$, taken on by $\operatorname{sgn}_{p}(x)=\left(\operatorname{sgn} p_{1}(x), \cdots, \operatorname{sgn} p_{m}(x)\right)$, $x=\left(x_{1}, \cdots, x_{n}\right) \in R^{n}$, is less than $2^{m}$ if $m \geqq 9 n \log _{2} d$.

COROLlaRY 2. If $m \geqq 20 n \log _{2} d$, the number of sign sequences $s$ which differ from any $\operatorname{sgn}_{p}(x)$ in at most $m / 13$ places is less than $2^{m}$.

2. Applications to nonlinear approximation. Let $G$ be a compact space, and let $L$ be the linear space of real continuous functions on $G$ with the supremum norm. Let $F$ be a compact family of functions in $L$. Define $w_{m}$ as the largest number for which there exist points $u_{1}, \cdots, u_{m}$ in $G$ such that for any sign sequences $s=\left(s_{1}, \cdots, s_{m}\right)$ there is an $f$ in $F$ so that $s_{i} f\left(u_{i}\right) \geqq w_{m}, i=1, \cdots, m$.

THEOREM 2. If $S$ is a subset of $L$ depending polynomially of degree $d$ on $n$ parameters, and if $S$ approximates $F$ to within less than $w_{m}$, then $n \log _{2} d \geqq m / 9$.

Proof. Each $P(x)\left(S=\left\{P(x): x \in R^{n}\right\}\right)$ is a function on $G$; let $p_{i}(x)$ be its value at $u_{i}, i=1, \cdots, m$. If $m \geqq 9 n \log _{2} d$, there is an $s$ such that $\operatorname{sgn}_{p}(x) \neq s$ for any $x$. Let $\left(s_{1}^{*}, \cdots, s_{m}^{*}\right)$ be such an $s$, and choose $f$ in $F$ so that $s_{1}^{*} f\left(u_{i}\right) \geqq w_{m}$. The distance from $f$ to $S$ is at least $w_{m}$, contradiction.

This same technique can be extended to give a proof of the more general theorem of Vitushkin on approximation in the supremum norm by piecewise rational functions. 
Corollary 3. If $F$ is the family of functions in $\operatorname{Lip}_{1}[0,1]$ which vanish at zero, $S$ cannot approximate $F$ to within less than $1 /\left(19 n \log _{2} d\right)$.

There is a similar extension of known results in the case of approximation in the $L^{p}$ norm (see [1, Theorem 5]). As an example, let $G$ be a unit cube in $R^{j}$. Let $F$ be the real valued functions $f(u)$ on $G$ such that $|f(u)| \leqq 1$ and $|f(u+t)-f(u)| \leqq\|t\| \alpha$, \|\| being the Euclidean norm of $R^{j}$. Let $S$ be a subset of $L^{1}(G)$ (the measure ordinary Lebesgue measure) which depends polynomially of degree $d$ on $n$ parameters.

THEOREM 3. S cannot approximate $F$ to within less than $c /\left(n \log _{2} d\right)^{\alpha / j}$ in the $L^{1}(G)$ norm, where $c$ is a constant depending on $j$ and $\alpha$.

The proof is similar to that of Theorem 2. The stronger Corollary 2 is used in place of Corollary 1 , and one passes from $S$ to the polynomials $p_{i}(x)$ by integrals rather than by point evaluation.

\section{Special results on partitions by real varieties.}

TheOREM 4. A real plane curve of order $n$ divides the affine plane into at most $C_{n, 2}+n+1$ components. This bound is achieved if and only if the curve consists of $n$ straight lines in general position.

The proof is based on Harnack's theorem relating the number of circuits of a real curve to its genus, and on the local topology of real algebraic curves.

THEOREM 5. There is a number $C_{n}$ such that if $m>C_{n} d$, a regular configuration of $m$ real hypersurfaces in $R^{n}$, each of order not more than $d$, cannot partition $R^{n}$ into as many components as can md hyperplanes.

Here "regular" means that at a point in the intersection of any $j$ of the $m$ hypersurfaces the tangent hyperplanes to the intersecting hypersurfaces exist and are linearly independent. The proof is like that of Theorem 1 and relies on Harnack's theorem for certain estimates.

\section{REFERENCES}

1. G. G. Lorentz, Lower bounds for the degree of approximation, Trans. Amer. Math. Soc. 97 (1960), 25-34.

2. O. A. Oleinik and I. G. Petrovsky, On the topology of real algebraic surfaces, Izv. Akad. Nauk Ser. Mat. 13 (1949), 389-402.

3. H. S. Shapiro, Some negative theorems of approximation theory, Mich. Math. J. 11 (1964), 211-217.

4. A. G. Vitushkin, On multidimensional variations, Gosudarstv. Izdat. Tekhn.Teor. Lit., Moscow, 1955.

5. - Estimation of the complexity of the tabulation problem, Gosudarstv. Izdat. Fiz.-Mat. Lit., Moscow, 1959. 